\title{
A MODIFIED TRIPLE TEST-CROSS ANALYSIS TO TEST AND ALLOW FOR INADEQUATE TESTERS
}

\author{
J. L. JINKS and D. S. VIRK \\ Department of Genetics, University of Birmingham
}

Received 24.i.77

\begin{abstract}
SUMMary
When the population under investigation consists of highly inbred lines the full triple test-cross of Kearsey and Jinks (1968) supplemented by the selfed progenies of the population allows unambiguous and independent tests for epistasis and the adequacy of the pure-breeding testers, $L_{1}$ and $L_{2}$. This can also be achieved by supplementing the simplified triple test-cross of Jinks, Perkins and Breese (1969) with the selfed progenies of the $L_{1 i}$ and $L_{2 i}$ families.

If the $L_{1}$ and $L_{2}$ testers prove to be inadequate due to the presence of common loci, modifications of the analyses are proposed which correct the resulting biases in the genetical components of variation.
\end{abstract}

\section{InTRODUCTION}

FOR populations of pure-breeding lines the triple test-cross design of Kearsey and Jinks (1968) can be simplified by omitting the $F_{1}$ tester $\left(L_{3}\right)$ and hence the $L_{3 i}$ families and substituting the pure-breeding lines $\left(P_{i}\right)$ themselves in the test for epistasis (Jinks, Perkins and Breese, 1969). This is a perfectly satisfactory alternative if the pure-breeding testers $\left(L_{1}\right.$ and $\left.L_{2}\right)$ differ at all $k$ loci at which the population of inbred differs. If, however, $L_{1}$ and $L_{2}$ differ only at $k_{2}=k-k_{1}$ loci where $k_{2}<k$ and are homozygous at $k_{1}$ loci for the same alleles, the test for epistasis is no longer unambiguous and the estimates of the additive and dominance components of variation are biased (Virk and Jinks, 1977). In order to test and allow for such biases a modification of the simplified triple test-cross analysis is suggested in this paper.

\section{Experimental DESIGNS}

The following two experimental designs provide independent tests for epistasis and for the adequacy of the $L_{1}$ and $L_{2}$ testers and also allow the biases due to any inadequacy of $L_{1}$ and $L_{2}$ to be removed and their magnitudes estimated.

(a) The full triple test-cross design in which two extreme pure-breeding lines and the $F_{1}\left(L_{3}\right)$ are used as testers to produce $3 n$ progeny families $\left(L_{1 i}, L_{2 i}\right.$ and $\left.L_{3 i}\right)$ along with the $n$ parental pure-breeding lines grown in a replicated block design.

(b) Alternatively, for naturally self-fertilising crops it may be more convenient to raise progenies produced by selfing the $L_{1 i}$ and $L_{2 i}$ crosses rather than producing the $L_{3 i}$ crosses so that the $L_{1 i}$ and $L_{2 i}$ crosses and their selfs along with the $n$ parental lines and the two testers $L_{1}$ and $L_{2}$ are raised in a replicated block design. 


\section{Analysis}

It is convenient to divide the analysis into two parts, the one concerned with the tests for epistasis and the adequacy of the testers and the other with the estimation of unbiased genetical components when the testers are inadequate.

\section{(i) Tests for epistasis and adequacy of testers}

The simple additive-dominance model can be unsatisfactory cither because of the prcsence of non-allelic interactions or because the testers are inadequate. For each of the (a) and (b) designs we now have two separate tests for the simple model which when applied simultaneously can differentiate between thesc causcs. For design (a) the two tests are the same as those described by Kearsey and Jinks (1968) and Jinks et al. (1969), based on the significance of the variances of (i) $L_{1 i}+L_{2 i}-2 L_{3 i}$ and (ii) $L_{1 i}+L_{2 i}-$ $\bar{P}_{i}$ over the $i=1$ to $n$ inbred lines. If design (b) is used, test (i) is equivalent to the $C$ scaling test of Mather, namely $4 L_{1 i s}-2 L_{1 i}-\bar{P}_{i}-L_{1 s}$ or $4 L_{2 i s}-$ $2 L_{2 i}-\bar{P}_{i}-L_{2 s}$ for the $L_{1}$ and $L_{2}$ testers, respectively, where $s$ denotes the family produced by selfing the $i=\mathrm{l}$ to $n \mathrm{~L}_{1 i}$ and $\mathrm{L}_{2 i}$ families. This test can be applied to each set of progeny families separately and the deviations tested against the pooled error variances of the family means (see Mather and Jinks, 1971). Alternatively an analysis of variance may be performed for the following statistical comparisons:

$\begin{array}{lccrrrrr}\text { Test for } & L_{1 i s} & L_{2 i s} & L_{1 i} & L_{2 i} & \bar{P}_{i} & L_{1 s} & \bar{L}_{2 s} \\ L_{1 i} \text { progenics } & 4 & 0 & -2 & 0 & -1 & -1 & 0 \\ L_{2 i} \text { progcnics } & 0 & 4 & 0 & -2 & -1 & 0 & -1\end{array}$

The block $\times$ comparison item or the pooled within-family variances may provide the estimate of the corresponding error variance depending upon the field design. For each pair of loci displaying non-allelic interactions, the $\mathrm{C}$ scaling test will equal $-[1]-2[i]$ in a quarter of the progeny families of each tester.

Test (ii) can be applied independently on $L_{1 i}$ and $L_{1 i s}$, and $L_{2 i}$ and $L_{2 i s}$ progeny families. They can, however, be combined by employing an extension of Cavalli's scaling test (Mather and Jinks, 1971).

On the basis of tests (i) and (ii) four situations can arise in practice cach with its own interpretation. These are:

(a) Neither (i) nor (ii) are significant

This means that there is no detectable epistasis and the testers are adequate. Alternatively the testers may be inadequate but there is no dominance and hence the estimates of the paramcters are unbiased. Therefore the standard triple test-cross analysis of Jinks et al. (1969) can be used to test the significance and to obtain the estimates of the various parameters.

(b) (i) is non-significant and (ii) is significant

There is no epistasis but the testers are inadequate becausc they sharc common alleles. 'The biases arising from the latter must then be removed by the procedure described later.

(c) (i) is significant and (ii) is non-significant

This is trivial in that it could only arise because the two tests differ in their sensitivities to epistasis. There is also the possibility, though highly 
unlikely, of the two causes of failure of the simple model, namely epistasis and the inadequacy of the testers, having effects which cancel out in test (ii).

(d) Both (i) and (ii) are significant

Epistasis is present and we cannot therefore be sure whether the testers are or are not adequate. Any estimates of the genetical parameters will be biased by non-allelic interactions and possibly by inadequate testers also. We could remove any bias from the latter by proceeding with the estimation of the parameters as for (b).

\section{(ii) Estimation of unbiased components}

For situation (b) where failure of the simple model is attributable solely to inadequate testers the following procedure is proposed for obtaining unbiased estimates of the genetical components.

(i) Design (a)

For each set of $\bar{L}_{1 i}, L_{2 i}, \bar{L}_{3 i}$ and $\bar{P}_{i}$ families we can make the following orthogonal comparisons:

$\begin{array}{cccccc}\text { Comparison } & \bar{L}_{1 i} & \bar{L}_{2 i} & \bar{L}_{3 i} & \bar{P}_{i} & \text { Component } \\ 1 & 1 & 1 & 1 & 0 & \sigma_{s}^{2} \\ 2 & 1 & -1 & 0 & 0 & \sigma_{d}^{2} \\ 3 & 0 & 0 & 0 & 1 & \sigma_{p}^{2}\end{array}$

The first comparison replaces the variance of $\bar{L}_{1 i}+L_{2 i}$ as suggested by Jinks and Perkins (1970) for estimating $\sigma_{s}^{2}$ while comparison 2 is that originally suggested by Kearsey and Jinks (1968) for computing $\sigma_{d}^{2}$. The third comparison which estimates $\sigma_{p}^{2}$ will always give unbiased estimates of additive genetic component $(D)$ since it is independent of the testers used. The genetical expectations of the components of variance obtainable from these comparisons for any number of loci with arbitrary allele frequencies but with alleles independently distributed among the inbred lines will be:

$$
\begin{aligned}
\sigma_{s}^{2} & =\sum_{i=1}^{k} u v d_{i}^{2}+\sum_{i=1}^{k_{1}} u v h_{i}^{2}-2 r_{1} \sum_{i=1}^{k_{2}} u v d_{i} h_{i} \\
& =\frac{1}{4} D_{k}+\frac{1}{4} H_{k_{1}}-\frac{1}{2} F_{k_{1}} \\
\sigma_{d}^{2} & =\sum_{i=1}^{k_{2}} u v h_{i}^{2}=\frac{1}{4} H_{k_{2}} \\
\sigma_{p}^{2} & =\sum_{i=1}^{k} u v d_{i}^{2}=D_{k}
\end{aligned}
$$

Two further statistics which are not independent of these three are essential for the estimation of the unbiased parameters. These are:

$$
\begin{gathered}
\sigma^{2}\left(\bar{L}_{1 i}+\bar{L}_{2 i}-\bar{P}_{i}\right)=\frac{1}{9}\left[\sum_{i=1}^{k_{1}} 4 u v h_{i}^{2}\right]=\frac{1}{9} H_{k_{1}} \\
\operatorname{Cov}\left(\bar{L}_{1 i}+\bar{L}_{2 i}\right)\left(\bar{L}_{1 i}-\bar{L}_{2 i}\right)=-r_{2} \sum_{i=1}^{k_{2}} u v d_{i} h_{i}=-\frac{1}{4} F_{k_{2}}
\end{gathered}
$$

where $r_{1}$ and $r_{2}$ are the degrees of association of alleles of like effect (see Virk 
and Jinks, 1977 for details). The only other statistic $\sigma^{2}\left(L_{1 i}+L_{2 i}-2 \tilde{L}_{3 i}\right)$, which is orthogonal to the first three is, of course, an estimate of error variation only, in the situation of no epistasis under discussion.

The unbiased estimate of the dominance component of variation $(H)$ and covariance between additive and dominance effects can be obtained by adding the respective effects of the $k_{1}$ and $k_{2}$ loci as follows:

Dominance component, $H_{k}=H_{k_{1}}+H_{k_{2}}=4 \sigma_{d}^{2}+9 \sigma^{2}\left(\bar{L}_{1 i}+L_{2 i}-\bar{P}_{i}\right)$

Covariance, $F_{k}=F_{k_{1}}+F_{k 2}=\sigma_{p}^{2}+9 \sigma^{2}\left(\bar{L}_{1 i}+L_{2 i}-\bar{P}_{i}\right)-4$ Cov sums/ $\operatorname{diffs}-4 \sigma^{2}$

The standard errors of these parameters can be computed from the linear relationships of the statistics involved but since there are tests of significance for all the basic $\sigma^{2}$ 's and the covariance on which they are based there will in general be little necessity for computing them.

Alternatively, weighted least square cstimates of the components of variation can be obtained by using the reciprocals of the expected variances of these statistics as weights (see Mather and Jinks, 1971). If this procedure is used, additional statistics can be obtained from the pooled within family variance $\left(\sigma_{w}^{2}\right)$ of $L_{3 i}$ progenies and the variance between $L_{3 i}$ family mcans. These have the expectations:

$$
\sigma_{w \mathrm{~L}_{3 i}}^{2}=\sum_{i=1}^{k_{2}} u v\left(d_{i}^{2}+h_{i}^{2}\right)=\frac{1}{4} D_{k_{2}}+\frac{1}{4} H_{k_{2}}
$$

and

$$
\begin{aligned}
\sigma_{\dot{L}_{3 i}}^{2} & =\sum_{i=1}^{k} u v d_{i}^{2}+\sum_{i=1}^{k} u v h_{i}^{2}-2 r_{1} \sum_{i=1}^{k_{1}} u v d_{i} h_{i} \\
& =\frac{1}{4} D_{k}+\frac{1}{4} H_{k}-\frac{1}{2} F_{k_{1}}
\end{aligned}
$$

(ii) Design (b)

The expectations of $\sigma_{s}^{2}, \sigma_{i l}^{2}$ and the covariance of sums and differences computed from the selfed $L_{1 i}$ and $L_{2 i}$ progenics remain unchanged for the additive $(D)$ gene cffects while the cocfficients of the dominance $(H)$ and $F$ cornponents are reduced by $\frac{1}{4}$ and $\frac{1}{2}$, respectively for each generation of selfing. After making allowance for these changes we can obtain unbiased estimates of the genetical components by utilising the comparisons outlined for design (a) which do not use $L_{3 i}$ familics. The analysis of sums, differences and the covariance between them, in this situation, remains unaltered from that described by Jinks et al. (1969). We can therefore have two independent estimates of the parameters from the $L_{1 i}$ and $L_{2 i}$ and $L_{1 i s}$ and $L_{2 i s}$ sets of families.

For the weighted least square estimation the pooled within-family $\left(\sigma_{w}^{2}\right)$ variances of the $L_{1 i s}$ and $L_{2 i s}$ families yields an additional statistic which equals $\frac{1}{4} D_{k}+\frac{3}{8} H_{k}$. This statistic also provides for the direct cstimation of the unbiased dominance component $\left(H_{k}\right)$ since $D_{k}$ is obtainable from $\sigma_{p}^{2}$.

Both designs also provide for the estimation of the dominance $\left(H_{k}\right)$ component of variation by computing the deviations of each $L_{1 i}$ or $L_{2 i}$ 
family mean from its parental mean and performing an analysis of variance for the following statistical comparisons:

$\begin{array}{lcccrrc}\text { Comparison } & \bar{L}_{1 i} & L_{2 i} & \ddot{P}_{i} & \bar{L}_{1 s} & \bar{L}_{2 s} & \begin{array}{c}\text { expected value } \\ \text { of } \sigma^{2}\end{array} \\ \text { 1. For } L_{1 i} \text { progenies } & 2 & 0 & +1 & -1 & 0 & \frac{1}{36} H_{k} \\ \text { 2. For } L_{2 i} \text { progenies } & 0 & 2 & -1 & 0 & -1 & \frac{1}{36} H_{k}\end{array}$

The corresponding error variance for these comparisons can be obtained from the block interactions or from the pooled within-family variances depending upon the experimental design. A joint comparison of $I$ and 2 will only estimate the dominance effects of the $k_{1}$ common loci between the $L_{1}$ and $L_{2}$ testers and it is therfore not recommended. Because of its small coefficient this estimate of $H_{k}$ is relatively unreliable.

\section{Discussion}

If we combine the $3 n$ progeny families of a full triple test-cross investigation of a population of $n$ pure-breeding lines with the $P_{i}$ selfed families of the $n$ pure-breeding lines themselves, two tests for the presence of epistasis can be carried out. One of these, the test of Kearsey and Jinks (1968), is unambiguous and applicable to any population. It is not invalidated if the common loci $k_{1}>0$ and $k_{2}<k$ and it will always detect epistasis for the noncommon $k_{2}$ loci. The other test (Jinks et al., 1969) applies solely to purebreeding populations and it is an unambiguous test for epistasis only if $k_{1}=0$ and $k_{2}=k$. Under all other circumstances this test may be significant even when no epistasis is present because it is sensitive to the presence of common loci. Discrepancies between these two types of tests can, therefore, be used to detect the presence of common loci between the $L_{1}$ and $L_{2}$ testers.

Any test which unambiguously detects epistasis in all circumstances can be substituted for the test of Kearsey and Jinks (1968). For self-fertilising species in which it is easier to produce progenies by selfing $L_{1 i}$ and $L_{2 i}$ families than to produce $L_{3 i}$ families it can be replaced by the $\mathrm{C}$ scaling test of Mather (see Mather and Jinks, 1971).

Having demonstrated the inadequacy of the $L_{1}$ and $L_{2}$ testers due to the presence of common alleles at some loci the biases to which this normally leads (Virk and Jinks, 1977) can be avoided by following alternative procedures for estimating the genetical parameters. These procedures provide estimates of the dominance $(H)$ and $F$ components separately for the $k_{1}$ and $k_{2}$ loci at which $L_{1}$ and $L_{2}$ have common and non-common alleles respectively. Unfortunately, the price we pay for these estimates is the loss of one of the major advantages of the triple test-cross analysis, namely, uncorrelated estimates which are known with equal precision. Nevertheless, because most of the statistics used in these alternative procedures are still the same as those of the standard triple test-cross analysis the estimates vary less in precision and are less correlated than those that would be obtained from most, if not all, alternative designs.

Because it is a relatively simple analysis and requires fewer crosses than the original triple test-cross the simplified version of the triple test-cross design is becoming increasingly popular and in many instances detailed data are being collected and analysed for several quantitative traits simultaneously. The basic requirement of phenotypically extreme testers is 
being violated for some of the traits since the frequency of adequate testers is only $2^{k-1}$ out of $\left[2^{k}\left(2^{k}+1\right)\right] / 2$ possible combinations of randomly paired pure-breeding testers. Of these $2^{k-1}$ pairs only one will be completely associated and therefore, recognisable as phenotypic extremes, while all others will vary in their degree of gene dispersion. It follows, therefore, that there is quite a high probability of the testers being inadequate for any traits rccorded in the experiment other than that for which the testers werc selected as the phenotypic extremes. How high this probability is will depend largely upon the extent of the correlation betwecn the additional traits and that used for choosing the extreme testers. The proposed modified analysis permits the use of data from the additional traits without leading to biased estimates and misleading conclusions and therefore adds to the gencral range of situations in which the triple test-cross may be used. This modification, however, should not be regarded as a satisfactory alternative to the selcetion of adequate testers before the commencement of the experiment.

Acknowledgments.-These are due to the Royal Commission for the Exhibition of 1851 for financial support to D. S. V. during these investigations.

\section{ReFERENCES}

JINKS, J. L., AND prRkins, f. м. 1970. A general method for the detection of additive, doninance and epistatic components of variation. III. $F_{2}$ and backcross populations. Heredily, 25, 419-429.

JINKS, J. L., PERKINS, J. M., AND BREESE, E. L. 1969. A general method of detecting additive, dominance and epistatic variation for metrical traits. II. Application to inbred lines. Heredity, 24, 45-57.

KE.ARSFY, M. J., AND JINKS, J. L. 1968. A general method of detecting additive, dominance and epistatic variation for metrical traits. 1 . Theory. Heredity, 22, 97-103.

MATIER, K., AND JiNks, J. I. 1971. Biometrical Genetics, 2nd edition. Chapman and Hall, l.ondon.

VIRK, v. S., AND JINKS, J. L. 1977. The consequences of using inadequate testers in the simplified triple test-cross. Heredity, $38,237-251$. 